\title{
Flare Size
}

National Cancer Institute

\section{Source}

National Cancer Institute. Flare Size. NCI Thesaurus. Code C112283.

The size of the area of redness that forms around the site of an antigenic challenge to the skin. 